\title{
KardiaTool: An Integrated POC Solution for Non-invasive Diagnosis and Therapy Monitoring of Heart Failure Patients*
}

\author{
Evanthia E. Tripoliti, Member, IEEE, Georgia S. Karanasiou, Pinelopi Ioannidou, Petros \\ Toumpaniaris, Yorgos Goletsis, Member, IEEE, Joan Baussels, Senior Member, IEEE, Tommaso \\ Lomonaco, Norman Pfeiffer, Raphaël Anasthase, Aliki Tsopela, Boris Leekens, Katerina K. Naka, \\ Jean-Christophe Lourme, Pietro Salvo, Trifon Liakopoulos, Jake Jordan, Joseph Gallagher, \\ Abdelhamid Errachid, and Dimitrios I. Fotiadis, Senior Member, IEEE
}

\begin{abstract}
The aim of this work is to present KardiaTool platform, an integrated Point of Care (POC) solution for noninvasive diagnosis and therapy monitoring of Heart Failure (HF) patients. The KardiaTool platform consists of two components, KardiaPOC and KardiaSoft. KardiaPOC is an easy to use portable device with a disposable Lab-on-Chip (LOC) for the rapid, accurate, non-invasive and simultaneous quantitative assessment of four HF related biomarkers, from saliva samples. KardiaSoft is a decision support software based on predictive modeling techniques that analyzes the POC data and other patient's data, and delivers information related to $\mathrm{HF}$ diagnosis and therapy monitoring. It is expected that identifying a source comparable to blood, for biomarker information extraction, such as saliva, that is cost-effective, less invasive, more convenient and acceptable for both patients and healthcare professionals would be beneficial for the healthcare community. In this work the architecture and the functionalities of the KardiaTool platform are presented.
\end{abstract}

\section{INTRODUCTION}

Heart Failure (HF) is a life-threatening disease, causing a significant economic burden on healthcare systems across the globe due to its high prevalence, the extended hospitalization requirements and the recurrent readmission of the patients [1]. More specifically, the European Heart Failure Association reports that 23 million people experience HF worldwide, while 3.6 million people are newly diagnosed with HF every year in

*Research supported by the KardiaTool project that has received funding from the European Union's Horizon 2020 research and innovation program under the grant agreement No 768686.(http://www.kardiatool.eu/)

E.E Tripoliti, G.S. Karanasiou, P Ioannidou, and P. Toumpaniaris are with the Department of Biomedical Research, Institute of Molecular Biology and Biotechnology, FORTH, Ioannina, GR 45110, Greece, (email: etripoliti@gmail.com, penelopeioannidou@gmail.com, petros.toumpaniaris@gmail.com, ).

Y. Goletsis is with the Department of Economics, University of Ioannina, GR 45110, Ioannina, Greece (email: goletsis@cc.uoi.gr).

J. Baussels is with the Instituto de Microelectrónica de Barcelona, IMBCNM (CSIC), Campus UAB, 08193 Bellaterra, Spain (email: joan.bausells@imb-cnm.csic.es).

T. Lomonaco is with the Department of Chemistry and Indurstrial Chemistry, University of Pisa, Italy (email: tommaso.lomonaco@unipi.it).

$\mathrm{N}$. Pfeiffer is with the Image Processing and Medical Engineering Department, Fraunhofer Institute for Integrated Circuits IIS, 91058 Erlangen, Germany (email: norman.pfeiffer@iis.fraunhofer.de).

R. Anasthase is with the BioTray, 82 Rue Cyprian 69100 Villeurbanne, France (email: r.anasthase@biotray.fr).

A. Tsopela is with the Micronit Microtechnologies B.V., Colosseum 15, 7521 PV Enschede, The Netherlands (email: aliki.tsopela@micronit.com).

B. Leekens is with the Interuniversitair Microelectronica Centrum (IMEC), Kapeldreef 75, 300, Leuven, Belgium (email: Boris.Leekens@imec.be).
Europe [2]. The costs related to HF account for approximately $2 \%$ of the total healthcare costs, while indirect socioeconomic effects of HF disease include increased morbidity, disrupt productivity, increased medical debt and premature mortality. The worldwide HF economic burden is estimated at $108 €$ billion per year [3].

The process of $\mathrm{HF}$ diagnosis is challenging and complicated. Even if the pathophysiology of HF has been understood in great extent by the medical community, there is a wide spectrum of potential clinical manifestations of $\mathrm{HF}$; signs and symptoms are often non-specific [4]. Additionally, therapy monitoring of $\mathrm{HF}$ patients is of critical value for healthcare professionals; the identification of subgroups of patients can lead to a more intensive follow-up when needed, help avoiding frequent re-hospitalizations or delay disease progression to end stage HF. Currently the majority of HF therapy monitoring models are not personalized and are unsuitable for clinical application. One has to agree that there is ample room for improving the usability of risk prediction tools and improve HF therapy monitoring.

New biomarkers and testing technologies, which can diagnose $\mathrm{HF}$, monitor therapy and provide information related to the subsequent risk for adverse events or mortality of the affected patients may drive this change. There are several cardiac related biomarkers that are referred to molecules

K.K. Naka is with the Michaelidion Cardiac Center, University of Ioannina, and 2nd Department of Cardiology, University of Ioannina, GR 45110, Ioannina, Greece (email: drkknaka@ gmail.com).

J-C. Lourme is with the ValoTec, Villejuif Bio Park, 1, Mail du Professeur Georges Mathé, 94800 Villejuif, France (email: jc.lourme@ valotec.com).

P. Salvo is with the CNR Istituto di Fisiologia Clinica Pisa, Italy (e-mail: pietro.salvo@ifc.cnr.it).

T. Liakopoulos is with the Enachip Inc, 1095 Cranbury South River Road, Suite \#1, Jamesburg, NJ 08831 USA (email: trifon@enachip.com).

J. Jordan is with the Department of Clinical and Experimental Medicine, University of Surrey, Guildford, GU2 7XH, Surrey, United Kingdom (email: jake.jordan@surrey.ac.uk).

J. Gallagher is with the gHealth Research Group, University College Dublin, National University Of Ireland, Belfield, Dublin, Ireland (email: jgallagher@ucd.ie).

A. Errachid is with the Université de Lyon, Institut de Sciences Analytiques (ISA) - UMR 5280, 5 rue de la Doua, 69100 Villeurbanne, France (e-mail: abdelhamid.errachid@univ-lyon1.fr).

D.I. Fotiadis is with the Department of Biomedical Research, Institute of Molecular Biology and Biotechnology, FORTH, Ioannina, Greece and the Dept. of Materials Science and Engineering, Unit of Medical Technology and Intelligent Information Systems, University of Ioannina, GR 45110 , Ioannina, Greece (phone: +302651009006 , fax: +302651008889 , e-mail: fotiadis@cc.uoi.gr) 
carried with diagnostic and prognostic information [5]. Point of Care (POC) devices provide a great opportunity for continuous monitoring of such biomarkers. The POC market is driven by technological advancements that facilitate provision of Micro-Nano-Bio Integrated Systems platforms (MNBS) which enhance the ability to detect, analyze and monitor biomarkers as evaluation end-points, in a fast, accurate, user-friendly and cost effective way [6]. POC testing devices are commercially available for myoglobin, CK-MB, cTnI, cTnT, and other markers [7-12]. From the POCs currently available in the market, i-STAT [8] excels because of its successful integration of the silicon chip technology with electrochemical principles. i-STAT micro fabricated miniaturized biosensors show good performance and diagnostic characteristics in terms of sensitivity, reproducibility, short analysis time, and small sample size requirement, however, it is an invasive method for biomarker analysis.

KardiaTool will provide significant advantages over already available POC devices. The innovation lies in the incorporation of four saliva biomarkers that have been proven relevant in a laboratory setting and bring a new promising concept for addressing the priority needs of the clinical arena. KardiaTool will provide the capability of simultaneously measuring the following four saliva biomarkers: (i) N-terminal pro b-type natriuretic peptide (NT-proBNP), (ii) Tumor Necrosis Factor -a (TNF-a), (iii) Interleukin- 10 (IL-10), (iv) Cortisol. The accurate detection and quantification of the selected saliva biomarkers can assist in the diagnosis, since specific high levels of the selected biomarkers is an indication for $\mathrm{HF}$. In addition, for patients with $\mathrm{HF}$, specific fluctuations of the selected biomarkers and their correlation, are indicative for the deterioration of the status of the patient, i.e. NYHA class change, prediction of adverse events, and thus potential need for therapy change [13-14, 16-20]. This will be achieved through the development of a novel MNBS platform incorporating the key enabling technologies in the field of advanced materials: (i) for the synthesis of magnetic nanoparticles (MNPs) and development of MNPs coatings, towards providing adequate physicochemistry and tailored surface properties, and (ii) for the development of the Lab-onChip LOC and POC.

KardiaTool will include new features to: (i) diagnose HF by detecting the disease at an early stage, when it is easier and less expensive to treat effectively, (ii) reduce the time, cost and failure of misdiagnosis, (iii) monitor therapy of HF patients and stratify them into groups of high and low risk for HF event (decompensation, mortality), and (iv) shift the emphasis in HF management from disease to wellness.

\section{KARDIATOOL PLATFORM}

\section{A. KardiaTool Architecture}

The concept of the KardiaTool platform is shown in Fig. 1. The main components of the KardiaTool platform are the KardiaPOC and KardiaSoft.

KardiaPOC is an easy to use portable POC device providing key information of four saliva biomarkers simultaneously: (i) NT-proBNP, (ii) TNF-a, (iii) IL-10, and (iv) Cortisol. KardiaPOC integrates a microfluidic system, a variety of sensors, actuators, Microelectromechanical systems (MEMS),
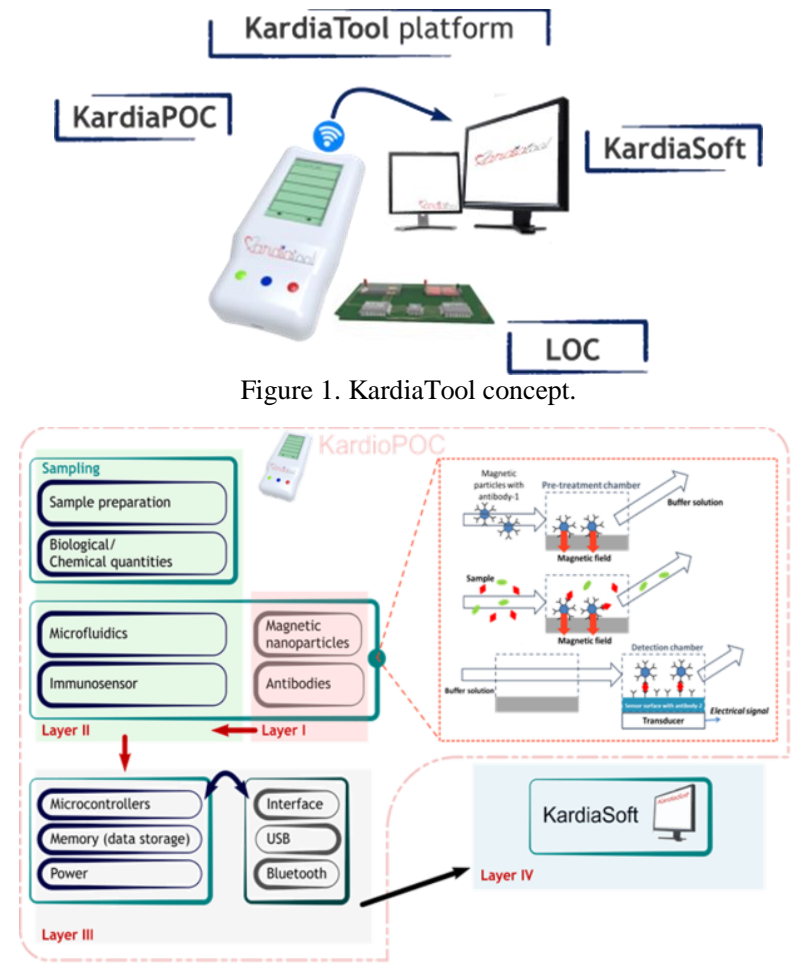

Figure 2. High level architecture of the KardiaTool platform.

micro-electronics, biochemical and functionalized MNPs, onto a disposable, low cost LOC.

KardiaSoft is a decision support software consisting of predictive models, correlating the output of the KardiaPOC device with additional heterogeneous data sources (e.g. clinical examinations, patient history, biological data, and other biomarkers) to allow for the extraction of patterns and rules towards efficient diagnosis and therapy monitoring for $\mathrm{HF}$, patient profiling-stratification and prognosis of $\mathrm{HF}$ adverse events.

The KardiaTool platform consists of the following layers (Fig. 2): (i) Layer I includes the MNPs and the antibodies, (ii) Layer II includes the saliva samples, the microfluidic system and the immunosensor component, (iii) Layer III includes the microcontroller, the memory and the power supply, communication interface, instrumentation of LOC, (iv) Layer $I V$ includes the KardiaSoft.

The process from saliva sampling to diagnosis and therapy monitoring consists of four steps: Step 1: The healthcare professional/nurse collects saliva from the patient and injects a sample into the microfluidic cartridge. The microfluidic cartridge is designed and fabricated in such a way that it integrates a flow control system with on-chip microfluidic valves and pumps. These elements are controlled through a pre-programmed sequence, by the controller integrated in the KardiaPOC. The sample is firstly pumped into the first microfluidic chamber where it is mixed with the functionalized MNPs. This first chamber is dedicated to magnetic beadsbased biomarkers capture for pre-concentration and purification, using planar coils for mixing and capturing cycles. After immunoreaction, the washing step with buffer solution will be implemented in order to remove absorbed biomolecules on the MNPs. The reacted MNPs will be dispensed in a precise manner to the second chamber. 
Electrochemical detection will be performed in this fully automated and miniaturized Field Effect Transistor (FET)immunosensor chip. Step 2: The healthcare professional/nurse loads the Ion Sensitive FET (ISFET) immunosensor array chip cartridges into the KardiaPOC device. Step 3: Analysis is performed and the concentration of each biomarker is displayed on the screen of the KardiaPOC device. Step 4: The biomarker results and the other patient and clinical data are automatically transferred to and analyzed by the KardiaSoft tool which then provides clinical information, software-based healthcare-related advice and support to assist the healthcare professionals in HF diagnosis and HF therapy monitoring.

\section{B. KardiaPOC}

The KardiaPOC device combines: (i) Microfluidic system, (ii) Nanocarriers, (iii) MEMS, (iv) Immunosensors, (v) Instrumentation.

Microfluidic system. A microfluidic cartridge consisting of a network of channels and chambers, as well as the microfluidic flow control elements, enabling precise fluid displacement for high reliability measurements will be developed. KardiaPOC microfluidic cartridge will be assembled to the LOC circuit board containing the magnetic and sensing chips. The architecture of the microfluidic system will contain the fluidic channels, sample preparation and sensing chambers, integrated sensors, flow actuation, reagent reservoirs (integrated blisters) and an injection system for the sample.

Nanocarriers. A new concept of nanocarriers consisting of MNPs functionalized with secondary antibodies for preconcentration of the HF biomarkers will be followed. Functionalized MNPs made of iron oxide ( $\left.\mathrm{Fe}_{3} \mathrm{O}_{4}-\mathrm{NPs}\right)$, which have attracted much attention in the fabrication of bio-sensing systems, due to their unique properties (super-paramagnetism, biocompatibility, signal amplification, etc.) will be developed. One of the novelties associated to KardiaTool platform is the "to and fro" movement of the functionalized MNPs in the front chamber that hosts the electromagnet. This is achieved by the on/off switching of the electromagnet that will attract and liberate the nanocollector MNPs. This shall permit that the loading of the MNPs becomes possible not only by diffusion but also by convection.

$M E M S$. Different types of coils with and without magnetic cores will be microfabricated with MEMS processing techniques on silicon wafers and will be tested for power limitations, performance and reliability within the microfluidic environment. More specifically, the following types of inductors will be evaluated: (i) square spiral inductor with air core, (ii) double spiral air core, (iii) single or double spiral with magnetic core, (iv) toroid with magnetic core. Finite Element Analysis of the coils will help to narrow down the designs that will be fabricated on silicon wafers for functionality tests.

Immunosensors. Detection of saliva biomarkers requires high detectability. The ultimate state-of-the-art FET designs will be used together with the MNPs to improve the electrochemical signal. Technologies for sample-pretreatment and pre-concentration chamber together with detection chamber will be integrated for the very first time in the same chip with maximized resolution and reliability in large volume scalable low cost devices.
Instrumentation. A new electronic circuit for the determination of the transconducatance of protein-modified HfO2 (Hafnium dioxide) ISFET will be designed and fabricated. Major objectives and features of the instrumentation will be: (i) small form factor applying Surface Mount Devices, in smallest sizes, (ii) low power consumption using selected components, (iii) onboard power and memory management, (iv) standardized communication interfaces and data transfer, (v) processing unit for system control and measurement analysis, (vi) various functional blocks for the system control of the biochemical sensor: initialization, cartridge detection, drivers for micro-pumps and micro-coils and, (vii) various functional blocks for the Electrochemical impedance spectroscopy measurement [15].

The mastering complexity of the aforementioned advanced technologies will provide numerous advantages in terms of KardiaPOC diagnostic speed and sensitivity.

\section{KardiaSoft}

KardiaSoft will be a multi-purpose and multifunctional computational tool that will be based on a variety of HF related data. The decision support processing is a critical component of KardiaSoft tool since it will provide valuable clinical information to the healthcare professionals and assist them in (i) diagnosis of $\mathrm{HF}$, (ii) profiling and stratification of $\mathrm{HF}$ patients, (iii) prediction of $\mathrm{HF}$ adverse events, and (iv) therapy monitoring, in real time. The machine learning techniques, that will be tested, include decision trees and rule based approaches for HF diagnosis, clustering algorithms for profiling and patient stratification, dynamic techniques, which take time dependencies into consideration, for progress prediction and complex regression techniques for estimating the time and probability of HF adverse events. Through the detection of changes in the output of the above mentioned functionalities (i-iii) therapy monitoring will be achieved.

\section{KardiaTool HF biomarkers}

Circulating levels of NT-proBNP are normally very low in healthy individuals. NT-proBNP levels are increased in HF and correlate well with ventricular wall stress and severity of HF [16]. With regard to the prognostic value of NT-pro-BNP for HF, a correlation between NT-pro-BNP and advanced HF is demonstrated [17]. The most important cytokines implicated in the progression of HF are TNF-a and IL-10 [18]. It is well established that high concentrations of TNF- $\alpha$ is observed in patients with $\mathrm{HF}$ and that these levels correlate with the patient's functional class. Patients with HF have a significant decrease in their serum level of IL-10 and increase in TNF-alpha and these levels change significantly with advanced NYHA class [19]. Serum levels of cortisol appear to be equally strong independent predictors of all-cause mortality risk in patients with systolic and non-systolic HF of any cause. The highest serum concentrations of cortisol identified a subgroup of HF patients with a particularly high mortality risk [20].

\section{E. KardiaTool proof of concept}

The evaluation of the KardiaTool platform will be conducted through a pre-clinical testing, a two stage clinical testing and health technology assessment (HTA) analysis. The pre-clinical testing aims to validate the accuracy of the measurements of the KardiaPoC device. Specifically, the 
validation will be carried out by comparing the KardiaPOC measurements with the analytical reference methods. Conventional immunoassay methods will be used, along with innovative ultra-high performance liquid chromatographic techniques coupled with high-resolution tandem mass spectrometric detectors. Samples will be collected from 10 patients with symptoms of HF and known HF (Group I), 20 subjects with symptoms (hypertension, obesity) but without HF (Group II) and 15 patients with acute HF (Group III). They will be used for the validation of the KardiaPOC measurements following well-defined Quality control and Quality assurance protocols.

The clinical testing consists of two stages: i) a small clinical testing for the design and development of the KardiaSoft tool, and ii) an early clinical testing for the validation of the KardiaTool platform. Information from 135 subjects for the first stage and 90 subjects for the second stage (45 subjects of Group I, 90 subjects of Group II, 90 subjects of Group III) will be collected within three clinical centers (University Of Ioannina Greece- UOI, Consiglio Nazionale Delle Ricerche Italy - CNR, University College Dublin Ireland - UCD). Through early clinical testing the accuracy in: (i) HF diagnosis and, (ii) HF therapy monitoring, to prevent relapses in patients with established diagnosis of $\mathrm{HF}$ and for early detection of HF acute decompensation, will be validated.

In order to provide evidence of the need of adopting the KardiaTool platform, a detailed and thorough HTA will be performed focusing on the safety, efficacy and effectiveness, social, ethical and economic aspects of this technology tool.

\section{DISCUSSION}

KardiaTool will develop a personalized MNBS platform and bring a new laboratory proven concept from the laboratory to clinic, addressing priority needs in healthcare diagnosis and therapy monitoring for patients with symptoms for HF and patients with known HF. This approach is expected to provide considerable improvement for healthcare delivery at the POC, the quality of life of patients and a reduction in healthcare costs. The integration of biosensors, microarrays, micropumps, microelectronics and magnetics and other mechanical and electrical components onto chips, results in rapid, highly sensitive and advanced HF biomarkers identification and detection in a quick, non-invasive and accurate way. KardiaTool possesses a radical new approach based on the development of pre-concentration and multiplex analysis, microfluidics and functionalized HfO2-ISFETs. This accessory will allow: (i) Real time assessment of patient parameters based on saliva analysis, (ii) Pre-concentration and multiplex analysis, allowing the co-occurrence analysis, (iii) Improved detection technology, (iv) In-project clinical implication. KardiaTool offers many advantages over centralized laboratory testing, such as small reagent volumes, rapid analysis, small size, low power consumption, reliability, robustness and user acceptability. Saliva sampling has advantages compared to blood and urine. It is an easy and non-invasive process requiring relatively simple instructions for collection and it possesses less complexity than other body substances. The analysis of the KardiaPOC measurements with other critical patient data, through KardiaSoft, will enable personalized decision making for effective management of those at risk of an HF event.

The challenge of KardiaTool is three-fold: (i) to translate a laboratory proven concept of a saliva biosensor into an MNBS platform, for HF diagnostics and therapy monitoring, at the point of care, whilst following Good Laboratory Practice [21], (ii) to validate the MNBS platform through pre-clinical and clinical testing, following Good Clinical Practice [21], and (iii) to industrialize the outcomes, following Good Manufacturing Practice [21] towards progress to marketization.

\section{REFERENCES}

[1] "Cardiovascular diseases statistics - Statistics Explained." [Online]. Available:http://ec.europa.eu/eurostat/statisticsexplained/index.php/Cardiovascular diseases statistics.

[2] M. R. Cowie, "The heart failure epidemic," Medicographia, 2012.

[3] C. Cook, G. Cole, P. Asaria, R. Jabbour, and D. P. Francis, "The annual global economic burden of heart failure," Int. J. Cardiol., vol. 171, no. 3, pp. 368-376, 2014.

[4] A. Alonso-Betanzos, V. Bolón-Canedo, G. R. Heyndrickx, and P. L. Kerkhof, "Exploring Guidelines for Classification of Major Heart Failure Subtypes by Using Machine Learning," Clin. Med. Insights Cardiol., vol. 9, no. Suppl 1, pp. 57-71, 2015.

[5] S.T. Sanjay, G. Fu, M. Dou, F. Xu, R. Liu, H. Qi, et al., "Biomarker detection for disease diagnosis using cost-effective microfluidic platforms," Analyst, vol. 140, pp. 7062-7081,2015.

[6] Cardiac marker - Diagnostic testing market." Available: http://www.marketresearch.com/product/sample-8769761.pdf.

[7] "RAMP ${ }^{\circledR}$ Reader | Response Biomedical." Available: http://responsebio.com/products/ramp-reader

[8] "i-STAT Handheld Blood Analyzer | Abbott Point of Care." Available: https://www.pointofcare.abbott/int/en/offerings/istat/istat-handheld.

[9] "cobas h 232 POC system." Available: http://www.cobas.com/content/internet/product/cobas/en/home/produc t/point-of-care-testing/cobas-h-232.html.

[10] "Samsung LABGEO PT10 | Samsung Business." Available: http://www.samsung.com/global/business/healthcare/healthcare/invitro-diagnostics/BCA-PT10/DE.

[11] "PATHFAST ${ }^{\circledR}$ - LSI Medience Corporation." Available: http://www.pathfast.com/.

[12] "Meritas ${ }^{\circledR} \quad$ Archives - Trinity Biotech." Available: http://www.trinitybiotech.com/area/meritas-en/.

[13] H. K. Gaggin and J. L. Januzzi, "Biomarkers and diagnostics in heart failure," Biochim. Biophys. Acta, vol. 1832, no. 12, pp. 2442-2450, Dec. 2013.

[14] N. Ansari, A. Hasan, and M. Owais, "A study of inflammatory markers and their correlation with severity, in patients with chronic heart failure." Biomedical Research, vol. 23, pp. 408-415, 2012.

[15] R. Pruna, et al., "A low-cost and miniaturized potentiostat for sensing of biomolecular species such as TNF- $\alpha$ by electrochemical impedance spectroscopy", Biosens Bioelectron., vol. 100, pp. 533-540. 2018.

[16] A.S. Maisel et al., "Rapid measurement of B-type natriuretic peptide in the emergency diagnosis of heart failure," N. Engl. J. Med., vol. 347, no. 3, pp. 161-167, Jul. 2002.

[17] L. Maries and I. Manitiu, "Diagnostic and prognostic values of B-type natriuretic peptides (BNP) and $\mathrm{N}$-terminal fragment brain natriuretic peptides (NT-pro-BNP)," Cardiovasc. J. Afr., vol. 24, no. 7, pp. 286289, 2013.

[18] S.D. Anker and S. von Haehling, "Inflammatory mediators in chronic heart failure: an overview," Heart, vol. 90, no. 4, pp. 464-470, 2004.

[19] A. Longo et al., "Highly Sensitive Electrochemical BioMEMS for TNF- $\alpha$ Detection in Human saliva: Heart Failure," Procedia Eng., vol. 168, pp. 97-100, 2016.

[20] G. Güder et al., "Complementary and incremental mortality risk prediction by cortisol and aldosterone in chronic heart failure," Circulation, vol. 115, no. 13, pp. 1754-1761, 2007.

[21] "Research Quality Association." Available: https://www.therqa.com/about/. 